\title{
Association between inhaled corticosteroids and upper respiratory tract infection in patients with chronic obstructive pulmonary disease: a meta- analysis of randomized controlled trials
}

Hong Chen ${ }^{1 *}\left(\mathbb{D}\right.$, Yulin Feng ${ }^{2}$, Ke Wang $^{2}$, Jing Yang ${ }^{2}$ and Yuejun Du ${ }^{1}$

\begin{abstract}
Background: We aimed to assess the association between inhaled corticosteroids (ICSs) and the risk of upper respiratory tract infection (URTI) in patients with chronic obstructive pulmonary disease (COPD).

Methods: PubMed, Embase, Cochrane Library and Clinical Trials.gov were searched from inception to October 2019. Randomized controlled trials (RCTs) of any ICSs vs control for COPD with reporting of URTI as an adverse event were included. The study was registered with PROSPERO prospectively (\#CRD42020153134).

Results: Seventeen RCTs (20,478 patients) were included. ICSs significantly increased the risk of URTI in COPD patients (RR, 1.13; 95\% Cl 1.03-1.24; $P=0.01$; heterogeneity: $P^{2}=7 \%$ ). Futher subgroup analyses suggested that shortterm use of ICSs increased the risk of URTI (RR, 1.29; 95\% CI 1.06-1.56; $P=0.01$; heterogeneity: $P^{2}=14 \%$ ) but not for long-term use (RR, 1.08; 95\% Cl 0.97-1.2; $P=0.14$; heterogeneity: $P^{2}=0 \%$ ). Short-term use of high-dose fluticasone increased the risk of URTI (RR, 1.33; 95\% Cl 1.03-1.71; $P=0.03$; heterogeneity: $\left.P^{2}=0 \%\right)$ but not for long-term use (RR, 1.12; 95\% Cl 0.97-1.29; $P=0.13$; heterogeneity: $P^{2}=50 \%$ ). Medium-dose (RR, 0.97; $95 \%$ Cl 0.71-1.32; $P=0.84$; heterogeneity: $\left.P^{2}=0 \%\right)$ and low-dose $\left(\mathrm{RR}, 1.39 ; 95 \% \mathrm{Cl} 0.92-2.1 ; P=0.12\right.$; heterogeneity: $\left.P^{2}=30 \%\right)$ fluticasone did not increase the risk of URTI regardless of duration. Neither mometasone (RR, 1.05; 95\% Cl 0.87-1.26; $P=0.61$; heterogeneity: $P^{2}=0 \%$ ) nor budesonide (RR, 1.08; 95\% Cl 0.77-1.5; $P=0.67$; heterogeneity: $P=46 \%$ ) increased the risk of URTI, regardless of dosage or duration.
\end{abstract}

Conclusions: Long-term use of ICSs does not increase the risk of URTI in patients with COPD. Short-term use of highdose fluticasone increases the risk of URTI in patients with COPD, but not mometasone or budesonide.

Keywords: Inhaled corticosteroids (ICS), Chronic obstructive pulmonary disease (COPD), Upper respiratory tract infection (URTI), Risk, Meta-analysis

\footnotetext{
* Correspondence: apple0831@126.com

'Department of Infectious Disease, Chengdu Second People's Hospital, No.

10 Qingyun South Street, Chengdu 610017, China

Full list of author information is available at the end of the article
}

(c) The Author(s). 2020 Open Access This article is licensed under a Creative Commons Attribution 4.0 International License, which permits use, sharing, adaptation, distribution and reproduction in any medium or format, as long as you give appropriate credit to the original author(s) and the source, provide a link to the Creative Commons licence, and indicate if changes were made. The images or other third party material in this article are included in the article's Creative Commons licence, unless indicated otherwise in a credit line to the material. If material is not included in the article's Creative Commons licence and your intended use is not permitted by statutory regulation or exceeds the permitted use, you will need to obtain permission directly from the copyright holder. To view a copy of this licence, visit http://creativecommons.org/licenses/by/4.0/. The Creative Commons Public Domain Dedication waiver (http://creativecommons.org/publicdomain/zero/1.0/) applies to the data made available in this article, unless otherwise stated in a credit line to the data. 


\section{Introduction}

Chronic obstructive pulmonary disease (COPD) is currently the third leading cause of death and disability worldwide [1-3]. Exacerbation is the major reason for hospital admission of patients with COPD and related to a significantly worse survival outcome [4-6]. Inhaled corticosteroids (ICSs) or combined with long-acting bronchodilators have been recommended to treat COPD patients with repeated exacerbations [1].

Although ICSs are generally considered to be relatively safe and well tolerated in patients, some adverse effects associated with ICSs have also been observed, such as the development of oropharyngeal candidiasis [6], adrenal suppression [7], diabetes [8], and pneumonia [9]. However, the association between ICSs and risk of upper respiratory tract infection (URTI) remains unclear, though URTI is the most common respiratory infection and also an important cause of exacerbation of COPD [10]. The large prospective study Toward a Revolution in COPD Health (TORCH) trial reported ICSs might increase the morbidity of URTI in COPD patients [11]. Moreover, other randomized controlled trials (RCTs) reported different or even contrary outcomes, and most of these studies were inadequate to detect significant difference between ICSs treatment groups and control groups [12-17].

Whether ICSs increase the risk of URTI in COPD patients may depend on duration, dosage and type of ICSs. Lack of safety evidence may result in insufficient use or over use of ICSs. Therefore, we conducted this metaanalysis of RCTs to assess the association between ICSs use and the risk of URTI in patients with COPD. We also aimed to clarify the contributions of medication details for the association, including duration, dosage level and type of ICSs.

\section{Methods}

\section{Study protocol}

This meta-analysis was conducted in accordance with the Preferred Reporting Items for Systematic Reviews and Meta-Analyses (PRISMA) recommendations [18]. And the study was registered with PROSPERO prospectively (\#CRD42020153134).

\section{Search strategy}

Two reviewers independently searched PubMed, Embase, Cochrane Library and Clinical Trials.gov for eligible articles from inception to May 10, 2019, and updated on October, 16, 2019. Both free words and MeSH terms referring to inhaled corticosteroid and the risk of URTI were used as search terms, including "Pulmonary Disease, Chronic Obstructive" OR "chronic obstructive pulmonary disease" OR "COPD" OR "airflow obstruction, chronic" OR "chronic airflow obstruction" OR "chronic obstructive airway disease" OR "chronic obstructive lung disease" OR "Bronchitis" OR "emphysema" AND "ICS" OR "inhaled corticosteroids" OR "fluticasone" OR "flunisolide" OR "budesonide" OR "beclomethasone" OR "triamcinolone" OR "mometasone" OR "ciclesonide". We also conducted a manual search using the reference lists of key articles.

\section{Eligibility criteria}

Eligible studies were identified through the PICOS criteria (participants, interventions, comparators, outcomes and study design) [18]. Inclusion criteria included: (1) patients with COPD; (2) The interventions included any type of inhaled corticosteroids, including ICSs alone or combined with long-acting bronchodilators; (3) nonICSs treatment as control, including placebo or other inhaled drugs of corticosteroid free; (4) only trials reporting data on URTI as the outcome were included; (5) Only RCTs were included. Exclusion criteria included: (1) non-RCTs, such as observational studies, case series and reviews; (2) non-English articles; (3) Patients with asthma or unknown diagnosis; (4) ICSs was used in both the treatment group and the control group.

\section{Data collection process}

Two investigators independently extracted relevant data from the included RCTs into standardized collection forms for the outcomes and evidence. Disagreements between the two investigators were resolved by discussion, and a third investigator was consulted if necessary. The corresponding authors were contacted when relevant data were not available.

\section{Risk of bias assessment and quality of evidence}

Two investigators independently performed the risk assessment using the Cochrane Collaboration risk of bias tool [19]. Any disagreements between the two investigators were resolved by discussion, and a third investigator was consulted if necessary. The included RCTs were assessed according to the following features (1) random sequence generation; (2) allocation concealment; (3) blinding of participants and personnel; (4) blinding of outcome assessment;(5) selective reporting; (6) incomplete outcome data; (7) other bias. Each item was assessed as low, unclear, or high risk of bias.

\section{Statistical analysis}

We performed meta-analyses for quantitative data synthesis using Revman Software (v.5.3, Cochrane Collaboration, London, UK). The weights of each study were estimated by Mantel-Haenszel method. We calculated the risk ratio (RR) and 95\% confidence interval (CI) for the risk of URTI. A two-tailed $p$ value $<0.05$ was set for statistical significance. Heterogeneity was assessed using 
the $\mathrm{I}^{2}$ test, with $\mathrm{I}^{2}>50 \%$ indicating a substantial heterogeneity [20]. A random-effect model would be used when a substantial level of heterogeneity was found, otherwise a fixed-effect model would be used.

\section{Subgroup analysis}

Subgroup analyses were conducted according to the following variables: (1) duration (long [ $\geq 6$ months] and short [ $<6$ months]); (2) dosage of ICSs [21] (high dose [defined as $>500 \mu \mathrm{g} /$ day of fluticasone propionate or equivalent], medium dose [defined as $>250-500 \mu \mathrm{g} /$ day of fluticasone propionate or equivalent] and low dose [defined as $100-250 \mu \mathrm{g} /$ day of fluticasone propionate or equivalent]); (3) type of ICSs, including fluticasone, mometasone, budesonide, and beclomethasone.

\section{Results}

\section{Study selection and study characteristics}

Figure 1 shows the study selection process. A total of 3011 references were identified after an initial search, and 17 RCTs [11-17, 22-31] including 20,478 patients were finally included in the meta-analysis. Of the 17 RCTs, 16 were multicenter, double-blind, randomized trials. These studies were published from 2002 to 2019, with population sizes ranging from 149 to 6184 participants. Duration of the trials ranged from 1 month to 36 months, with 10 trials $[11,12,15,16,24-27,29,30]$ longer than or equal to 6 months, and 7 trials $[13,14,17$, $22,23,28,31]$ shorter than 6 months. Eleven trials [11, $13,15-17,23,25,26,28-30]$ investigated a high-dose ICSs treatment, 8 trials $[12,16,22,24,27,29-31]$ investigated a medium-dose ICSs treatment, and 5 trials [12, $14,17,27,28]$ investigated a low-dose ICSs treatment. Fluticasone was evaluated in 10 trials $[11,13-15,17$, $22-24,26,28]$, mometasone in 4 trials $[16,25,29,30]$, budesonide in 3 trials [12, 27, 31], and beclomethasone in 1 trial [27]. Table 1 shows the main characteristics of the included studies.

\section{Risk of bias and quality of evidence}

All trials were assessed using the Cochrane Collaboration risk of bias assessment tool. RCTs with four or more features were considered to be of high quality. Seventeen RCTs were regarded as high quality according to the risk of bias assessment tool and were included in the meta-analysis. All studies had low risk of selective reporting bias. One study had high risk of blinding of participants and personnel bias, 1 study had high risk of blinding of outcome assessment bias, and 1 study had high risk of incomplete outcome data. One study had unclear risk of random sequence generation bias, 2 studies had unclear risk of allocation concealment bias, 1 study had unclear risk of incomplete outcome data bias and 7 studies had other bias (Fig. 2).

\section{Risk of URTI associated with ICSs}

All 17 RCTs with 20,478 patients reported URTI as an adverse event. The risk of URTI was 8.6\% (991 of 11,587 patients) in the ICSs treatment groups, 7.7\% (686 of 8891 patients) in the control groups, and $8.2 \%$ in all patients (1677 of 20,478 patients). Compared with nonICSs treatment, ICSs was associated with a significantly increased the risk of URTI in patients with COPD (RR, 1.13; 95\% CI 1.03-1.24; $P=0.01$; heterogeneity: $I^{2}=7 \%$ ) (Fig. 3).

\section{Risk of URTI associated with ICSs for different durations}

Long-term use of ICSs [11, 12, 15, 16, 24-27, 29, 30] (10 RCTs, 15,634 patients) did not increase the risk of URTI (RR, 1.08; 95\% CI 0.97-1.2; $P=0.14$; heterogeneity: $I^{2}=$ $0 \%)$, whereas short- term use of ICSs $[13,14,17,22,23$, $28,31]$ (7 RCTs, 4844 patients) was associated with a significantly increased the risk of URTI (RR, 1.29; 95\% CI 1.06-1.56; $P=0.01$; heterogeneity: $I^{2}=14 \%$ ) (Fig. 4).

\section{Risk of URTI associated with different doses of ICSs}

High-dose ICSs [11, 13, 15-17, 23, 25, 26, 28-30] treatment (11 RCTs, 12,930 patients) was associated with a significantly increased the risk of URTI (RR, 1.14; 95\% CI 1.02-1.27; $P=0.02$; heterogeneity: $I^{2}=0 \%$ ), but neither medium-dose ICSs (8 RCTs, 4849 patients) [12, 16, 22 , 24, 27, 29-31] (RR, 1.02; 95\% CI 0.82-1.27; $P=0.86$; heterogeneity: $I^{2}=0 \%$ ) nor low-dose ICSs (5 RCTs, 2699 patients) $[12,14,17,27,28]$ (RR, 1.25; 95\% CI 0.9-1.74; $P=0.18$; heterogeneity: $\left.I^{2}=1 \%\right)$ increased the risk of URTI (Fig. 5).

\section{Risk of URTI associated with high-dose ICSs for different durations}

Long-term use of high-dose ICSs [11, 15, 16, 25, 26, 29, 30] (7 RCTs, 12,916 patients) did not increase the risk of URTI (RR, 1.09; 95\% CI 0.98-1.22; $P=0.13$; heterogeneity: $I^{2}=$ $0 \%$, whereas short- term use of high-dose ICSs [13, 17, 23, 28] (4 RCTs, 2509 patients) was associated with a significantly increased the risk of URTI (RR, 1.31; 95\% CI 1.021.67; $P=0.03$; heterogeneity: $I^{2}=7 \%$ ) (Fig. 6).

\section{Risk of URTI associated with fluticasone}

Fluticasone [11, 13-15, 17, 22-24, 26, 28] (10 RCTs, 12, 547 patients) was associated with a significantly increased the risk of URTI for all dose groups (RR, 1.16; 95\% CI 1.04-1.3; $P=0.01$; heterogeneity: $I^{2}=27 \%$ ). Subgroup analyses suggested that high-dose fluticasone [11, $13,15,17,23,26,28]$ (7 RCTs, 9537patients) was associated with a significantly increased the risk of URTI (RR, 1.17; 95\% CI 1.03-1.32; $P=0.02$; heterogeneity: $I^{2}=$ $27 \%$ ), but neither medium-dose fluticasone (2 RCTs, 1505patients) [22, 24] (RR, 0.97; 95\% CI 0.71-1.32; $P=$ 0.84 ; heterogeneity: $\left.I^{2}=0 \%\right)$ nor low- dose fluticasone (3 


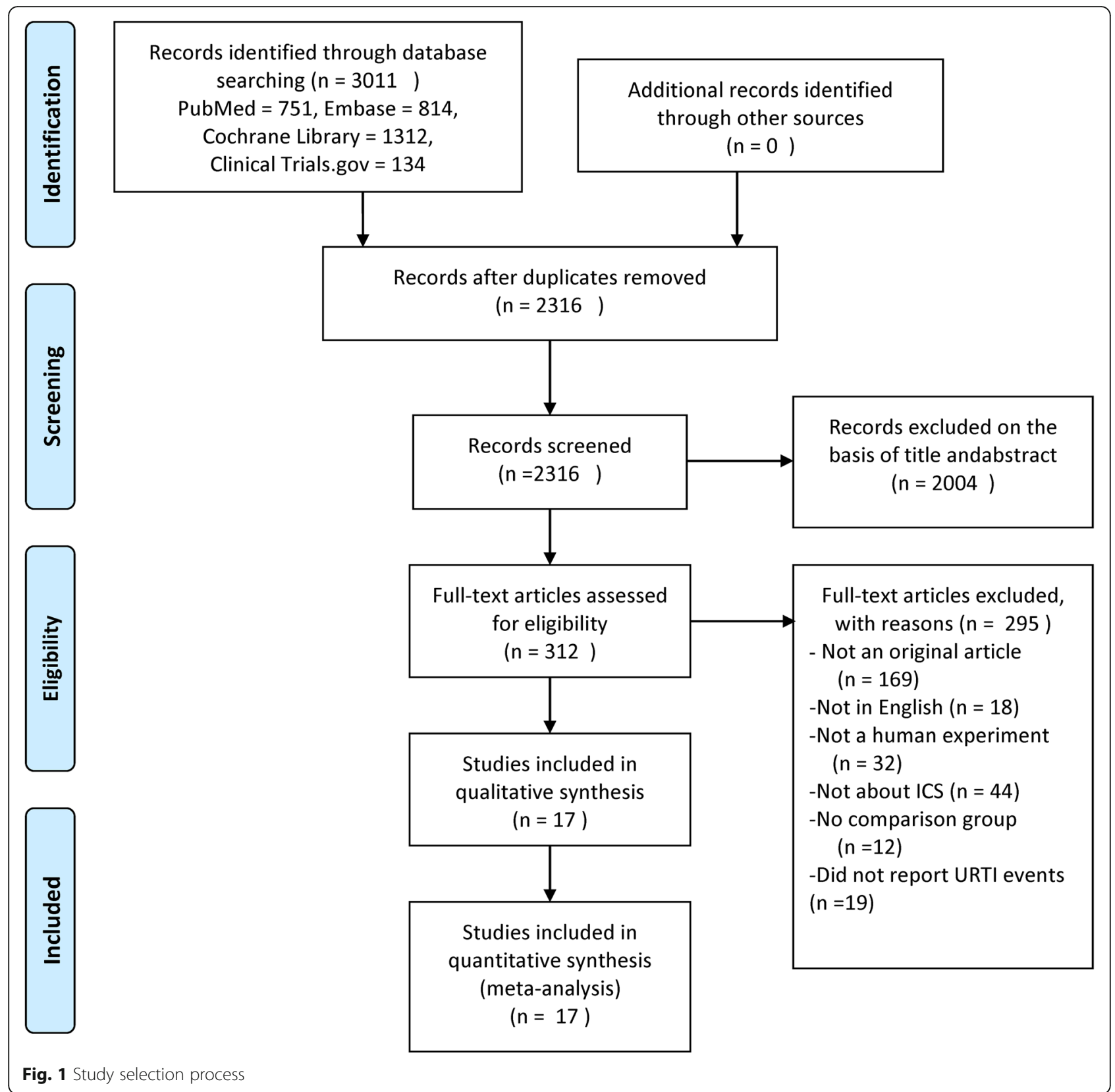

RCTs, 1964 patients) $[14,17,28](\mathrm{RR}, 1.39 ; 95 \%$ CI $0.92-$ 2.1; $P=0.12$; heterogeneity: $\left.I^{2}=30 \%\right)$ increased the risk of URTI. Moreover, long-term use of high-dose fluticasone (3 RCTs, 7503 patients) $[11,15,26]$ did not increase the risk of URTI (RR, 1.12; 95\% CI 0.97-1.29; $P=0.13$; heterogeneity: $\left.I^{2}=50 \%\right)$, whereas short-term use of high-dose fluticasone (4 RCTs, 2034 patients) was associated with a significantly increased the risk of URTI (RR, 1.33; 95\% CI 1.03-1.71; $P=0.03$; heterogeneity: $I^{2}=0 \%$ ) (Fig. 7).

\section{Risk of URTI associated with mometasone}

Mometasone [16, 25, 29, 30] (4 RCTs, 5413 patients) did not increase the risk of URTI for all dose groups (RR,
1.05; 95\% CI 0.87-1.26; $P=0.61$; heterogeneity: $I^{2}=0 \%$ ). Subgroup analyses suggested that neither high-dose mometasone (4 RCTs, 4521 patients) [16, 25, 29, 30] (RR, 1.06; 95\% CI 0.87-1.28; $P=0.57$; heterogeneity: $I^{2}=$ $0 \%)$ nor medium-dose mometasone (3 RCTs, 2692 patients) $[16,29,30]$ (RR, 0.98; 95\% CI 0.67-1.43; $P=0.93$; heterogeneity: $I^{2}=0 \%$ ) increased the risk of URTI (Fig. 8).

\section{Risk of URTI associated with budesonide}

Budesonide [12, 27, 31] (3 RCTs, 2518 patients) did not increase the risk of URTI (RR, 1.08; 95\% CI 0.77-1.5; $P=0.67$; heterogeneity: $I^{2}=46 \%$ ) (Fig. 9). 
Table 1 Characteristics of the 17 RCTs included in the meta-analysis of ICSs and risk of URTI

\begin{tabular}{|c|c|c|c|c|c|c|}
\hline Study & $\begin{array}{l}\text { Duration } \\
\text { (months) }\end{array}$ & $\begin{array}{l}\text { Mean Age } \\
\text { (years) }\end{array}$ & $\begin{array}{l}\text { FEV1 } \\
\text { (\%predicted) }\end{array}$ & Male (\%) & Interventions & NO. of URTI/Total \\
\hline \multirow[t]{2}{*}{ Mahler et al. [13] } & 5.5 & 63.5 & 40.8 & 66.0 & $\begin{array}{l}\text { T:FP } 500 \mu \mathrm{g} \text { bid or FSC (S } 50 \mu \mathrm{g} / F P \\
500 \mu \mathrm{g}) \mathrm{bid}\end{array}$ & $\mathrm{T}: 84 / 342$ \\
\hline & & & & & C: Placebo or S $50 \mu \mathrm{g}$ bid & C: $56 / 349$ \\
\hline \multirow[t]{2}{*}{ Hanania et al. [22] } & 5.5 & 63.8 & 41.8 & 63.2 & $\begin{array}{l}\text { T:FP } 250 \mu \mathrm{g} \text { bid or FSC (S } 50 \mu \mathrm{g} / \mathrm{FP} \\
250 \mu \mathrm{g}) \mathrm{bid}\end{array}$ & $\mathrm{T}: 40 / 361$ \\
\hline & & & & & C: Placebo or S $50 \mu \mathrm{g}$ bid & C: $42 / 362$ \\
\hline \multirow[t]{2}{*}{ Calverley et al. [11] } & 36 & 65.0 & 44.0 & 75.5 & $\begin{array}{l}\text { T:FP } 500 \mu \mathrm{g} \text { bid or FSC (S } 50 \mu \mathrm{g} / \mathrm{FP} \\
500 \mu \mathrm{g} \text { ) bid }\end{array}$ & T:309/3098 \\
\hline & & & & & C: Placebo or S $50 \mu \mathrm{g}$ bid & C: $277 / 3086$ \\
\hline \multirow[t]{2}{*}{ Zheng et al. [23] } & 5.5 & 66.2 & 47.0 & 89.2 & T:FSC (S $50 \mu \mathrm{g} / \mathrm{FP} 500 \mu \mathrm{g}$ ) bid & $\mathrm{T}: 32 / 297$ \\
\hline & & & & & C: Placebo & C: $14 / 148$ \\
\hline \multirow[t]{2}{*}{ Ferguson et al. [24] } & 12 & 65.0 & 32.8 & 55.0 & T:FP $250 \mu \mathrm{g}$ bid & $\mathrm{T}: 31 / 394$ \\
\hline & & & & & C: $S 50 \mu \mathrm{g}$ bid & C: $31 / 388$ \\
\hline \multirow[t]{2}{*}{ Calverley et al. [25] } & 12 & 65.1 & NR & 68.3 & T: MF $800 \mu \mathrm{g}$ qd or MF $400 \mu \mathrm{g}$ bid & T:164/616 \\
\hline & & & & & C: Placebo & C: $71 / 295$ \\
\hline \multirow[t]{2}{*}{ Anzueto et al. [26] } & 12 & 65.4 & 34 & 54.0 & T:FSC (S $50 \mu \mathrm{g} / F P 500 \mu \mathrm{g}$ ) bid & $\mathrm{T}: 41 / 394$ \\
\hline & & & & & C: Placebo & C: $30 / 403$ \\
\hline \multirow[t]{2}{*}{ Calverley et al. [27] } & 11 & 63.6 & 42.2 & 80.7 & $\begin{array}{l}\text { T:BDP/FM 200/12 } \mu \mathrm{g} \text { bid or } \\
\text { BUD/FM 400/12 } \mu \mathrm{g} \text { bid }\end{array}$ & $\mathrm{T}: 3 / 479$ \\
\hline & & & & & C: FM $12 \mu \mathrm{g}$ bid & C: 5/239 \\
\hline \multirow[t]{2}{*}{ Tashkin et al. [30] } & 6 & 59.8 & NR & 77.5 & $\begin{array}{l}\text { T: MF/FM 200/10 } \mu \mathrm{g} \text { bid or MF/FM } \\
400 / 10 \mu \mathrm{g} \text { bid or MF } 400 \mu \mathrm{g} \text { bid }\end{array}$ & T:19/634 \\
\hline & & & & & C: Placebo or FM $10 \mu \mathrm{g}$ bid & $C: 13 / 421$ \\
\hline \multirow[t]{2}{*}{ Tashkin et al. [16] } & 12 & 59.7 & 39.1 & 76.0 & $\begin{array}{l}\text { T: MF/FM 200/10 } \mu \mathrm{g} \text { bid or MF/FM } \\
400 / 10 \mu \mathrm{g} \text { bid or MF } 400 \mu \mathrm{g} \text { bid }\end{array}$ & $\mathrm{T}: 58 / 1351$ \\
\hline & & & & & C: Placebo or FM $10 \mu \mathrm{g}$ bid & C: $39 / 900$ \\
\hline \multirow[t]{2}{*}{ Doherty et al. [29] } & 6 & 59.6 & 38.6 & 75.2 & $\begin{array}{l}\text { T: MF/FM 200/10 } \mu \mathrm{g} \text { bid or MF/FM } \\
400 / 10 \mu \mathrm{g} \text { bid or MF } 400 \mu \mathrm{g} \text { bid }\end{array}$ & $\mathrm{T}: 39 / 717$ \\
\hline & & & & & C: Placebo or FM $10 \mu \mathrm{g}$ bid & C: $26 / 479$ \\
\hline \multirow[t]{2}{*}{ Sharafkhaneh et al. [12] } & 12 & 63.0 & 37.7 & 62.0 & $\begin{array}{l}\text { T:BUD/FM 320/9 } \mu \mathrm{g} \text { bid or BUD/FM } \\
\text { 160/9 } \mu \mathrm{g} \text { bid }\end{array}$ & $\mathrm{T}: 90 / 815$ \\
\hline & & & & & C: FM $9 \mu \mathrm{g}$ bid & C: $39 / 403$ \\
\hline \multirow[t]{2}{*}{ Boscia et al. [28] } & 1 & 57.9 & 49.8 & 46.3 & $\begin{array}{l}\text { T:FF/NI } 50 / 25 \mu \mathrm{g} \text { qd or FF/NI 100/25 } \\
\mu \mathrm{g} \text { qd or FF/ } 200 / 25 \mu \mathrm{g} \text { qd }\end{array}$ & $\mathrm{T}: 2 / 98$ \\
\hline & & & & & C: Placebo & C: $1 / 51$ \\
\hline \multirow[t]{2}{*}{ Vogelmeier et al. [15] } & 6 & 63.3 & 60.3 & 70.9 & T: FSC (S $50 \mu \mathrm{g} / F P 500 \mu \mathrm{g}) \mathrm{bid}$ & $T: 2 / 264$ \\
\hline & & & & & C: QVA149 110/50 $\mu \mathrm{g}$ qd & C: $7 / 258$ \\
\hline \multirow[t]{2}{*}{ Kerwin et al. [14] } & 5.5 & 62.5 & 48.3 & 66.6 & $\begin{array}{l}\text { T:FFNI } 50 / 25 \mu \mathrm{g} \text { qd or FFNI 100/25 } \\
\mu \mathrm{g} \text { qd or FF } 100 \mu \mathrm{g} \text { qd }\end{array}$ & $\mathrm{T}: 50 / 618$ \\
\hline & & & & & C: Placebo or VI $25 \mu \mathrm{g}$ qd & C: $19 / 412$ \\
\hline \multirow[t]{2}{*}{ Martinez et al. [17] } & 5.5 & 61.5 & 47.9 & 72.3 & $\begin{array}{l}\text { T:FFNI } 100 / 25 \mu \mathrm{g} \text { qd or FFNI } 200 / 25 \\
\mu \mathrm{g} \text { qd or FF } 100 \mu \mathrm{g} \text { qd or FF } 200 \mu \mathrm{g} \text { qd }\end{array}$ & $\mathrm{T}: 23 / 816$ \\
\hline & & & & & C: Placebo or VI $25 \mu \mathrm{g}$ qd & C: $14 / 408$ \\
\hline \multirow[t]{2}{*}{ Huang et al. [31] } & 2.8 & 64.1 & $N R$ & 86.4 & $\begin{array}{l}\text { T: BUD/FM 320/9 } \mu \mathrm{g} \mathrm{bid,} \mathrm{I} 40 \mu \mathrm{g} \text { qid } \\
\text { and T } 100 \mathrm{mg} \text { bid }\end{array}$ & T:4/293 \\
\hline & & & & & C:l $40 \mu \mathrm{g}$ gid and Th $100 \mathrm{mg}$ bid & C: $2 / 289$ \\
\hline
\end{tabular}

Abbreviations: ICSs Inhaled corticosteroids, URTI Upper respiratory tract infection, $F E V_{1}$ Forced expiratory volume in $1 \mathrm{~s}, T$ Treatment group, C Control group, NR Not reported, FP Fluticasone propionate, S Salmeterol, FSC Fluticasone propionate/salmeterol, $P$ Placebo, MF Mometasone furoate, BUD Budesonide, FM Formoterol, BDP Beclomethasone, QVA149 Indacaterol/glycopyrronium, FF Fluticasone furoate, VI Vilanterol, I Ipratropium, Th Theophylline, $Q D$ Once a day, BID Twice a day, QID Four times a day 

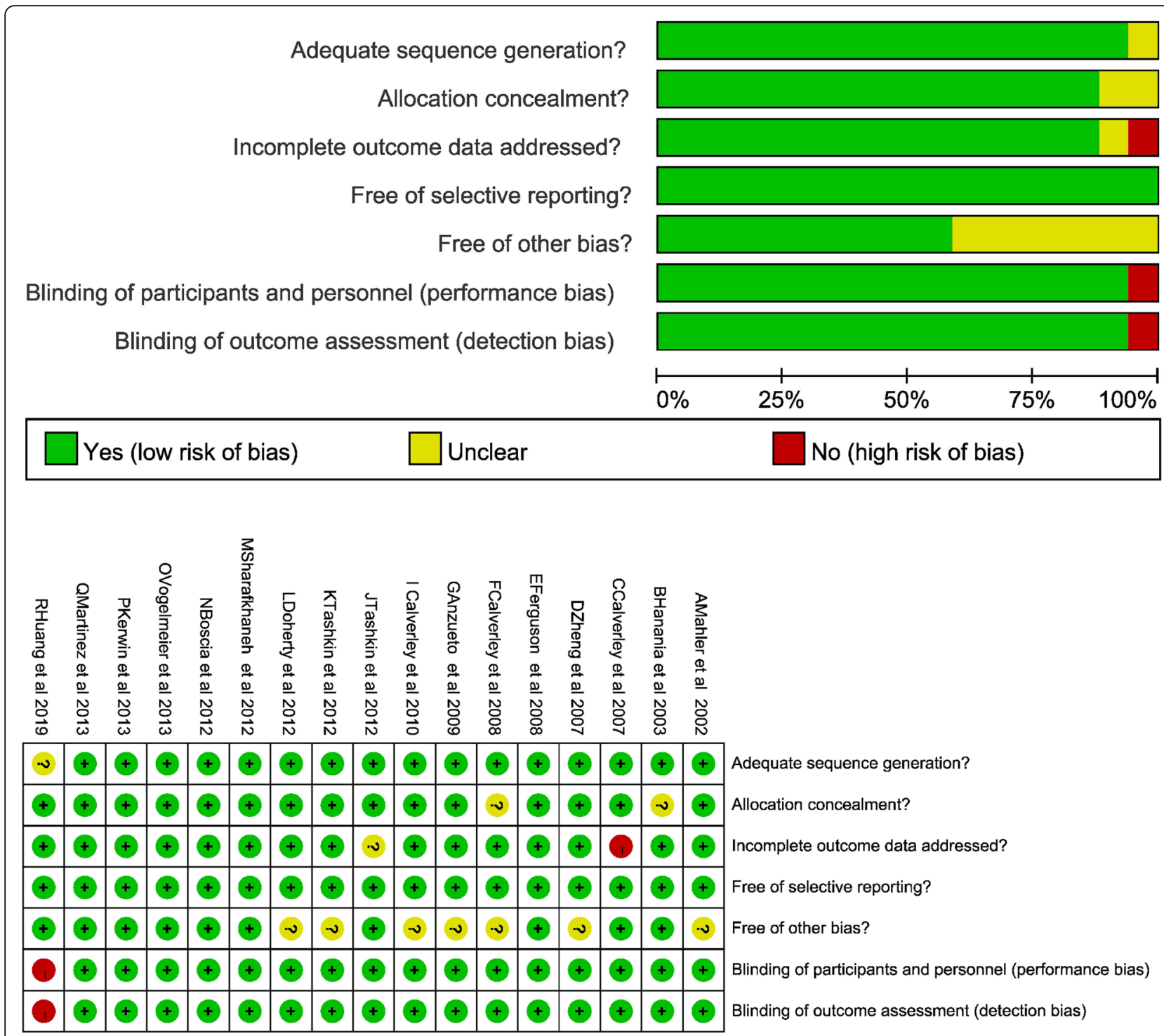

Fig. 2 Risk of bias of the included studies

\section{Discussion}

This meta-analysis of 17 multicenter RCTs (including 20,478 patients) suggested that short-term use of ICSs increased the risk of URTI but not for long-term use. Futher subgroup analyses revealed that only short-term use of high-dose fluticasone increased the risk of URTI but not for long-term use of high-dose fluticasone. Medium-dose and low-dose fluticasone did not increase the risk of URTI regardless of duration. Neither mometasone nor budesonide increased the risk of URTI, regardless of dosage or duration.

Exacerbation is common in patients with COPD affecting about $20 \%$ of patients with $40-45 \%$ of predicted $\mathrm{FEV}_{1}$ (1.3 events per year). Repeated exacerbations lead to worse survival outcome of patients [5]. Daily use of ICSs has been proved to decrease the frequency of exacerbations and improve quality of life in patients with $\mathrm{FEV}_{1}$ less than $50 \%$ predicted $[1,4]$. However, daily use of ICSs may cause drug-related adverse events, such as increased risks of fracture and infections [32, 33], but may not increase the risk of cardiovascular events [34]. URTI is the most common respiratory infections and also an important cause of exacerbation of COPD. Moreover, URTI can significantly reduce the quality of life in patients [10]. However, the association between ICSs and the risk of URTI remains unclear.

Our results suggested that use of ICSs was associated with a significantly increased the risk of URTI in COPD patients. However, further subgroup analyses suggested that only short-term use of ICSs significantly increased the risk of URTI but not for long-term use of ICSs in COPD patients. The result was unexpected. We 


\begin{tabular}{|c|c|c|c|c|c|c|c|c|c|c|}
\hline Study or Subgroup & \multicolumn{2}{|c|}{ ICSs } & \multicolumn{2}{|c|}{ Control } & Weight & $\begin{array}{c}\text { Risk Ratio } \\
\text { M-H. Fixed. } 95 \% \mathrm{Cl}\end{array}$ & \multicolumn{4}{|c|}{$\begin{array}{c}\text { Risk Ratio } \\
\text { M-H. Fixed. } 95 \% \mathrm{Cl}\end{array}$} \\
\hline Mahler et al 2002 & 84 & 342 & 56 & 349 & $7.3 \%$ & $1.53[1.13,2.07]$ & & & - & \\
\hline Hanania et al 2003 & 40 & 361 & 42 & 362 & $5.6 \%$ & $0.96[0.64,1.44]$ & & & t- & \\
\hline Calverley et al 2007 & 309 & 3098 & 277 & 3086 & $36.8 \%$ & $1.11[0.95,1.30]$ & & & $\mathbf{P}$ & \\
\hline Zheng et al 2007 & 32 & 297 & 14 & 148 & $2.5 \%$ & $1.14[0.63,2.07]$ & & & - & \\
\hline Ferguson et al 2008 & 31 & 394 & 31 & 388 & $4.1 \%$ & $0.98[0.61,1.59]$ & & & - & \\
\hline Calverley et al 2008 & 164 & 616 & 71 & 295 & $12.7 \%$ & $1.11[0.87,1.41]$ & & & $=$ & \\
\hline Anzueto et al 2009 & 41 & 394 & 30 & 403 & $3.9 \%$ & $1.40[0.89,2.19]$ & & & $=$ & \\
\hline Calverley et al 2010 & 3 & 479 & 5 & 239 & $0.9 \%$ & $0.30[0.07,1.24]$ & & & + & \\
\hline Tashkin et al 2012 & 19 & 634 & 13 & 421 & $2.1 \%$ & $0.97[0.48,1.94]$ & & & 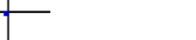 & \\
\hline Tashkin et al 2012 & 58 & 1351 & 39 & 900 & $6.2 \%$ & $0.99[0.67,1.47]$ & & & - & \\
\hline Doherty et al 2012 & 39 & 717 & 26 & 479 & $4.1 \%$ & $1.00[0.62,1.62]$ & & & - & \\
\hline Sharafkhaneh et al 2012 & 90 & 815 & 39 & 403 & $6.9 \%$ & $1.14[0.80,1.63]$ & & & 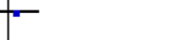 & \\
\hline Boscia et al 2012 & 2 & 98 & 1 & 51 & $0.2 \%$ & $1.04[0.10,11.21]$ & & & & \\
\hline Vogelmeier et al 2013 & 2 & 264 & 7 & 258 & $0.9 \%$ & $0.28[0.06,1.33]$ & & & - & \\
\hline Kerwin et al 2013 & 50 & 618 & 19 & 412 & $3.0 \%$ & $1.75[1.05,2.93]$ & & & 一 & \\
\hline Martinez et al 2013 & 23 & 816 & 14 & 408 & $2.5 \%$ & $0.82[0.43,1.58]$ & & 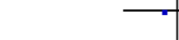 & - & \\
\hline Huang et al 2019 & 4 & 293 & 2 & 289 & $0.3 \%$ & $1.97[0.36,10.69]$ & & & & \\
\hline Total $(95 \% \mathrm{Cl})$ & & 11587 & & 8891 & $100.0 \%$ & $1.13[1.03,1.24]$ & & & $\beta$ & \\
\hline Total events & 991 & & 686 & & & & & & & \\
\hline $\begin{array}{l}\text { Heterogeneity: } \mathrm{Chi}^{2}=17 . \\
\text { Test for overall effect: } Z=\end{array}$ & $\begin{array}{l}f=16(P \\
2(P=0.0\end{array}$ & $\begin{array}{l}=0.37) \text {; } \\
\text { 1) }\end{array}$ & $1^{2}=7 \%$ & & & & 0.01 & $\begin{array}{l}0.1 \\
\text { Favours ICSs }\end{array}$ & ${ }^{1} \begin{array}{c}10 \\
\text { Favours Control }\end{array}$ & 100 \\
\hline
\end{tabular}

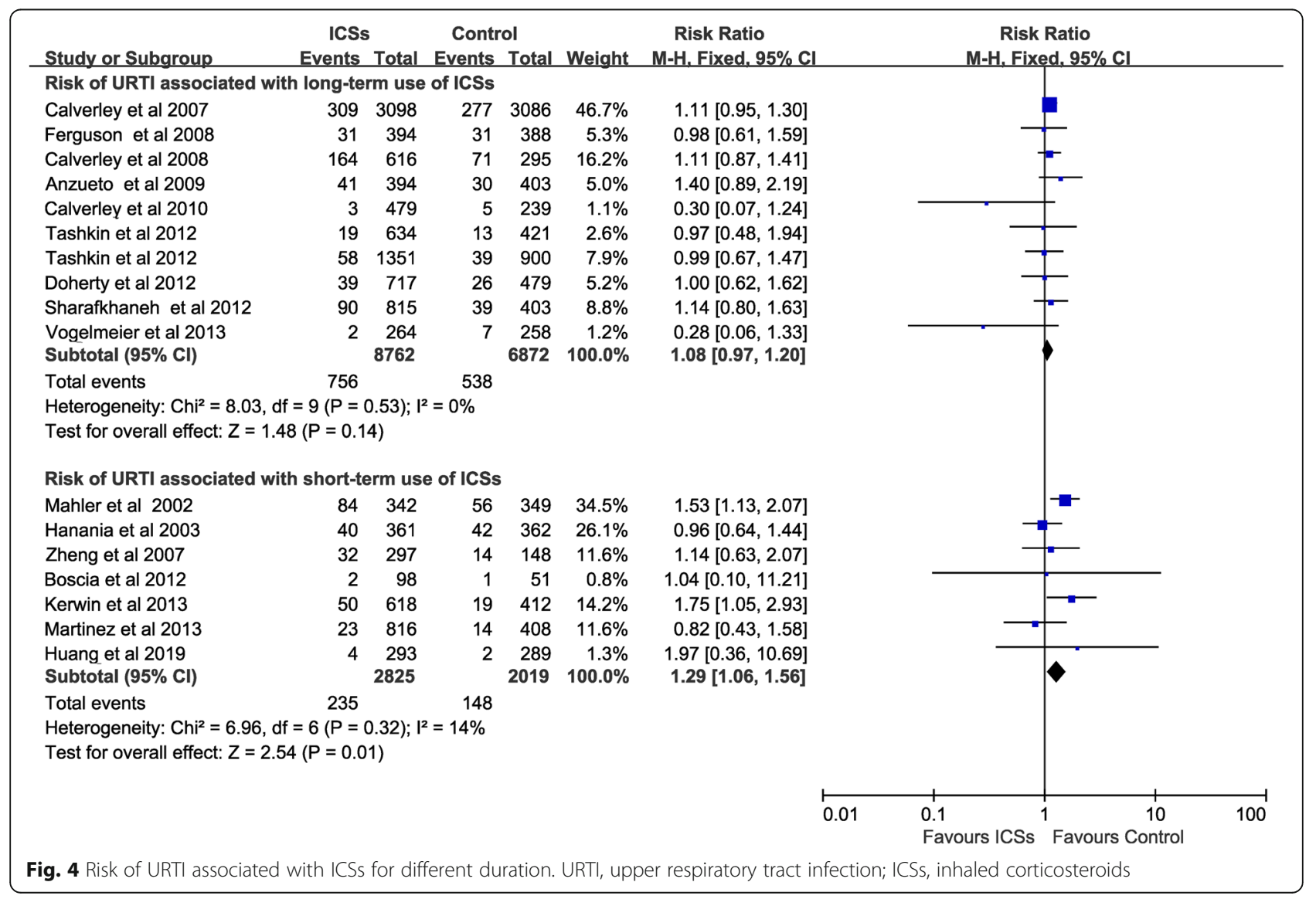




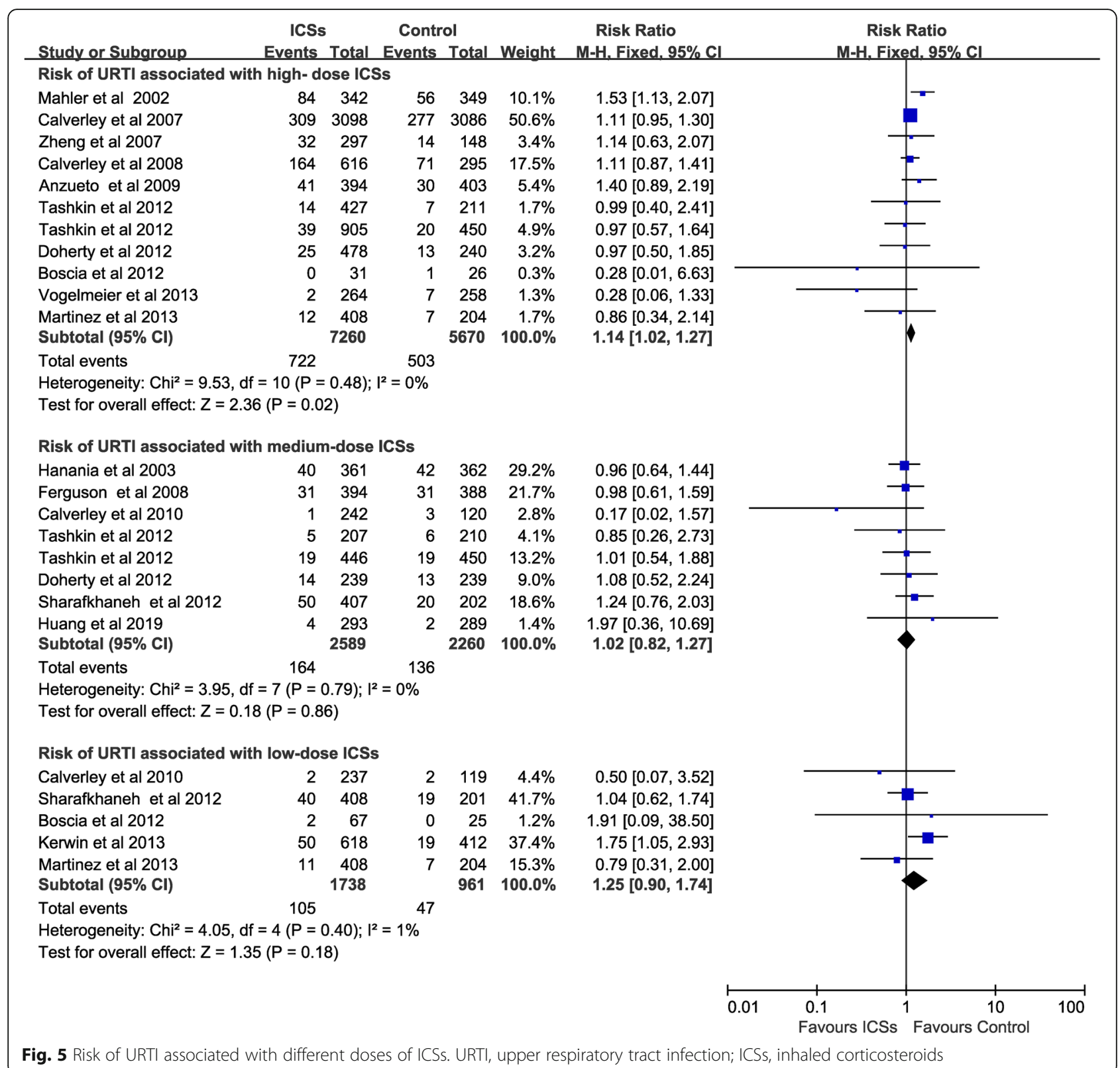

speculated that short-term use of ICSs could not effectively control the airway inflammation, whereas the immunosuppression effects of corticosteroids due to local high concentration might cause susceptibility to URTI in patients with COPD. On the contrary, long-term use of ICSs may improve health status of COPD patients by decreasing the frequency of exacerbations and improving clinical symptoms. In addition, the immune compensation mechanism of patients may play a role in resisting the immunosuppression of corticosteroid in the long term. A previous RCT conducted by Eichenhorn et al. may support our findings [35]. In their study, Eichenhorn et al. evaluated the effects of inhaled triamcinolone on adrenal function in 221 patients who were recruited from patients already enrolled in the Lung Health Study II, and found that use of triamcinolone ( $1200 \mu \mathrm{g}$ daily) for 3 years did not have suppression effects on adrenal function $[35,36]$.

Subgroup analyses were performed according to different dosage of ICSs. The results suggested that high-dose ICSs significantly increased the risk of URTI but not for medium- and low-dose ICSs. These findings may be explained by the possible dose-response effect of ICSs [7, 37]. In the further analysis, we found that only short-term use of high-dose ICSs was associated with a significantly increased the risk of URTI but not for long-term use of highdose ICSs. The results further supported that long-term use of ICSs did not increase ICS-related risk of URTI. 


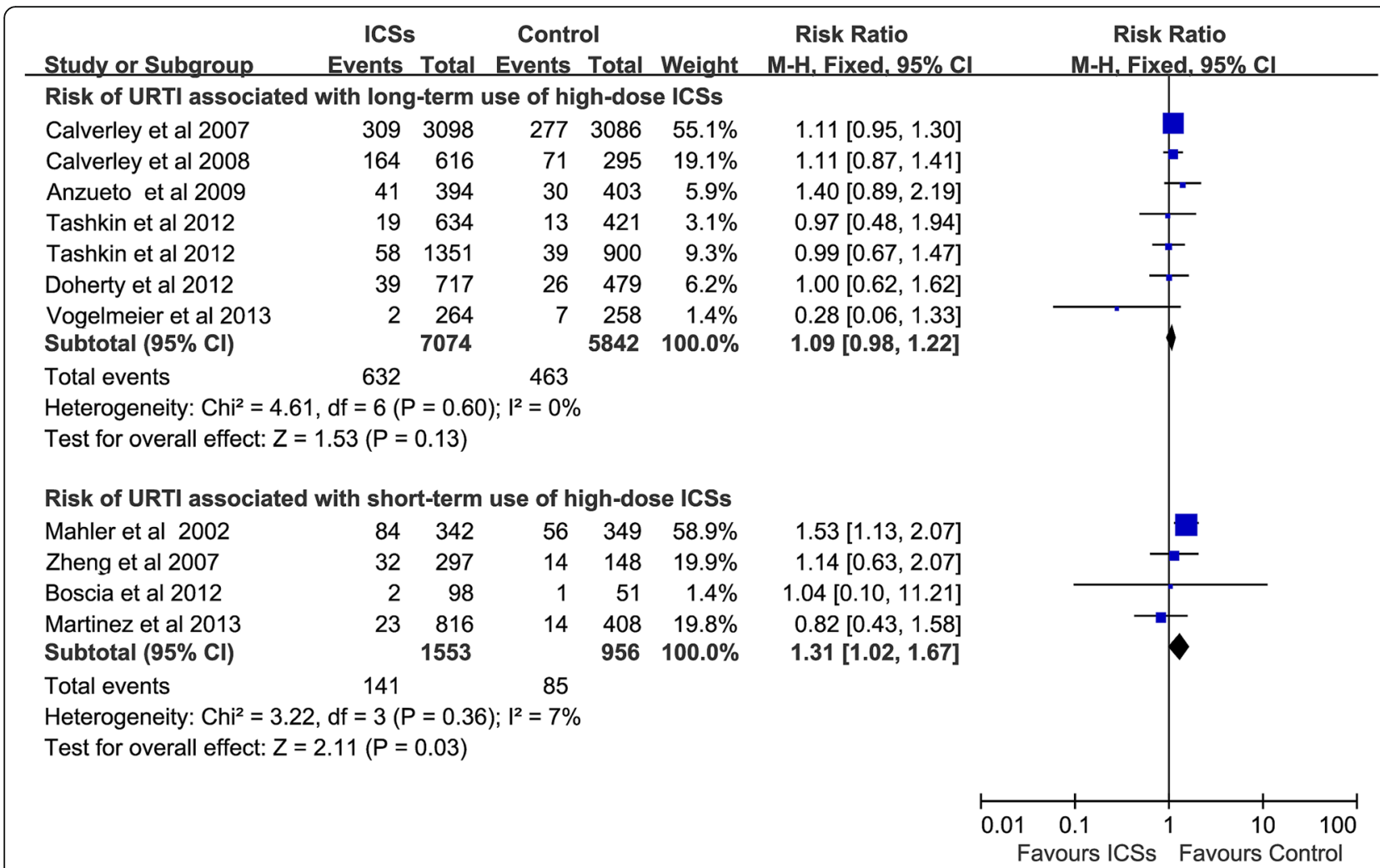

Fig. 6 Risk of URTI associated with high-dose ICSs for different durations. URTI, upper respiratory tract infection; ICSs, inhaled corticosteroids

We also conducted subgroup analyses according to type of ICSs. The results suggested that high-dose fluticasone was associated with a significantly increased risk of URTI but not for mometasone, budesonide, and medium- and low-dose fluticasone. Previous studies have reported that fluticasone may effectively suppress innate immunoresponse of alveolar macrophages, and the immunosuppressive effects of fluticasone might be tenfold greater than those of budesonide in human airways [38, 39]. Moreover, the included RCTs mainly explored medium- or low-dose budesonid rather than high-dose. Similarly, we also found that shortterm use of high-dose fluticasone was associated with a significantly increased the risk of URTI but not for long-term use of high-dose fluticasone. The results were consistent with the above results of the merged different types of ICSs.

Our results were not consistent with a previous metaanalysis conducted by Yang et al. in 2016 [40], which first reported that long-term use of ICSs may increase the risk of URTI in patients with COPD. However, that meta-analysis had limitations mainly because it did not include several large multicenter RCTs (including 3507 patients) published recently $[14,15,17,28,31]$. The smaller sample size of the previous meta-analysis may weaken the reliability and generalizability of the conclusion. Moreover, the previous meta-analysis failed to provide implication for clinical practice due to lack of subgroup analyses according to medication details including duration, dosage level and type of ICSs.

The strengths of our study were that we used a comprehensive search strategy and explicit inclusion criteria including 17 multicenter RCTs (20,478 patients), which met the requirements of sequential analysis. In addition, we conducted multiple subgroup analyses according to medication details including duration, dosage, and type of ICSs, that minimized the heterogeneity of pooled analyses and provided implications for clinical practice.

This study also had some limitations which mainly stemed from the quality of reported data. First, since some adverse events (such as URTI) were not the predefined outcomes and there were no homogeneous definitions among the clinical trials, these adverse events may be misclassified. This inherent methodological defect of clinical trials is one of the factors limiting the results of meta-analyses on drug safety. However, as most of the included trials in this meta-analysis were double blind, such misclassification would not have a substantial impact on the results of the meta-analysis, because the direction of bias may be toward the null. Second, the possibility of asthma-COPD overlap (ACO) in the study population may be one of the confounding factor of results. However, as a result of randomization, this confounding factor could not substantially affect the results 


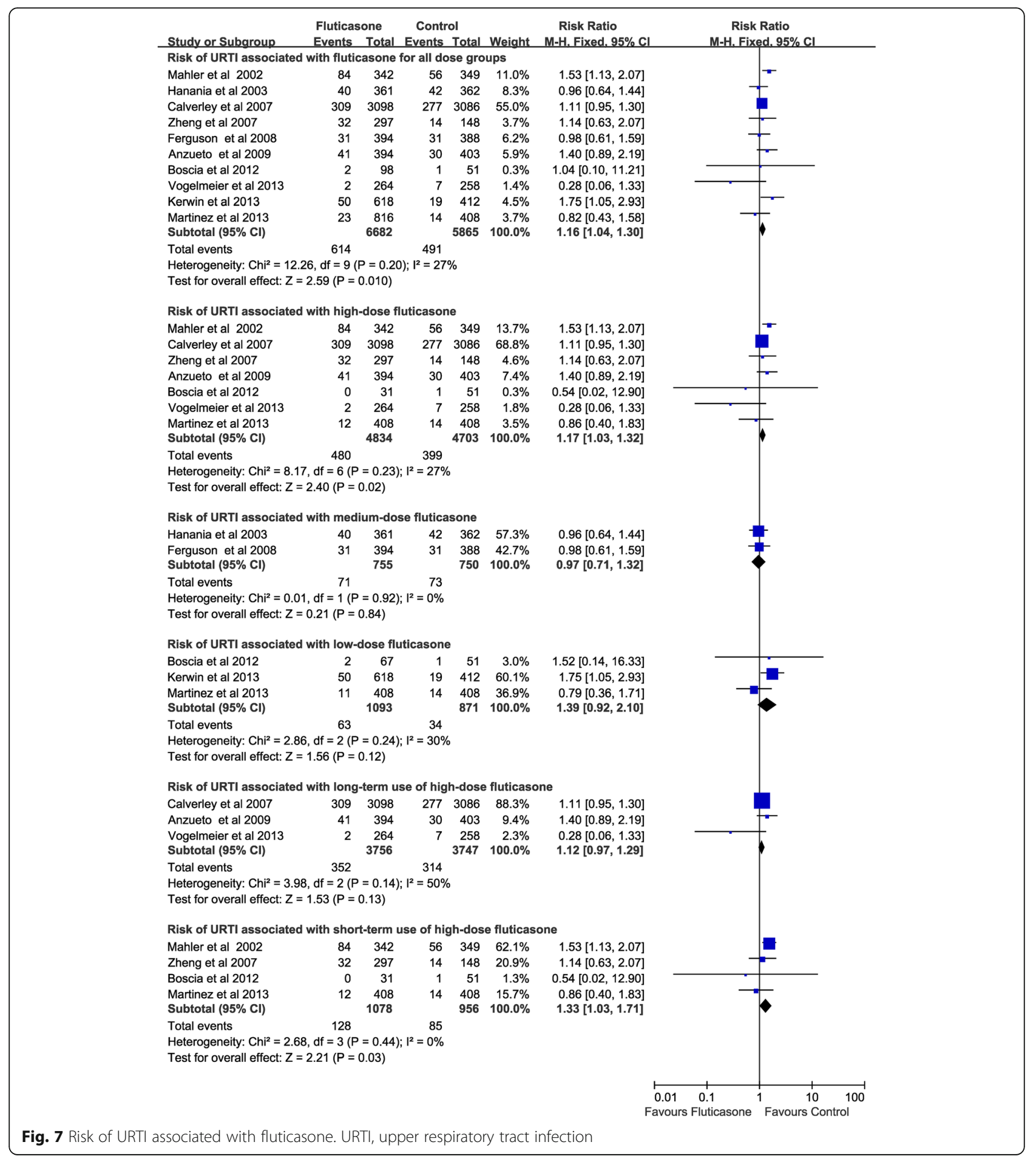

of the meta-analysis as ACO may be approximately evenly distributed between the ICSs treatment group and the control group. Third, further subgroup analyses could not be performed due to lack of some patient related information, such as eosinophil count, exacerbations in the past year, comorbidities, smoking status and preexisting ICSs use and this may be a confounding factor of results.

\section{Conclusions}

Long-term use of ICSs does not increase the risk of URTI in patients with COPD. Short-term use of high- 


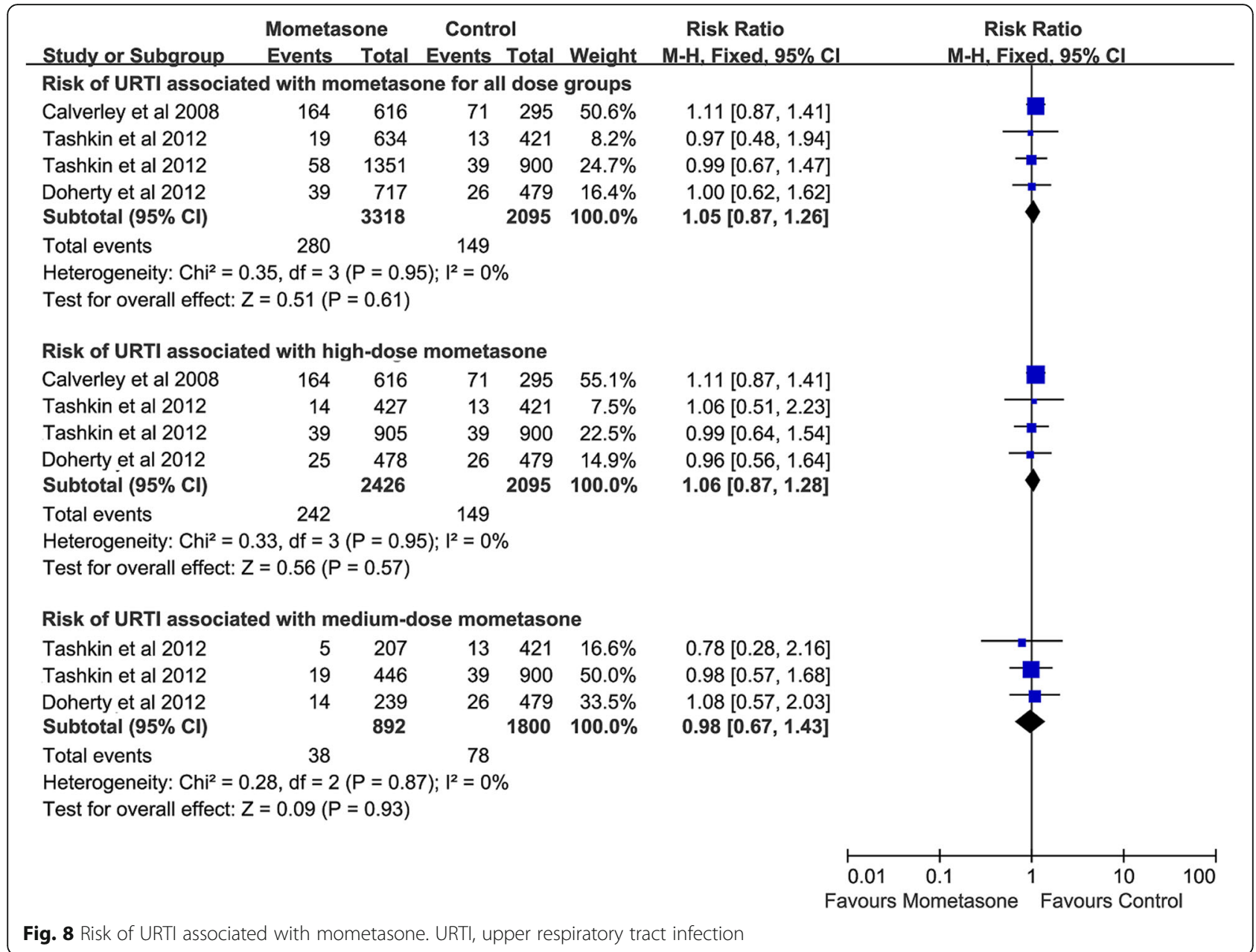

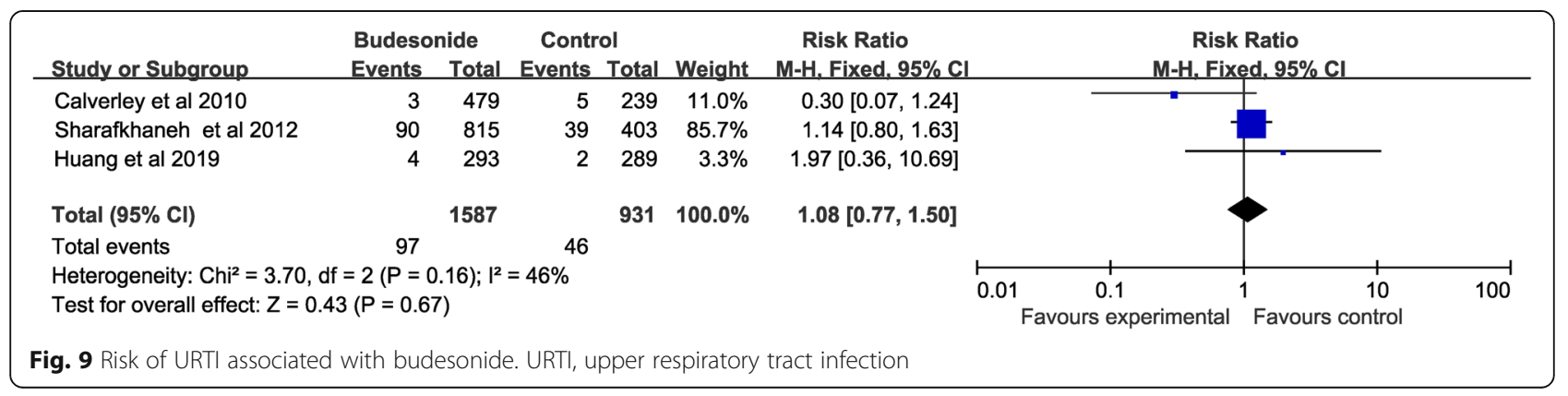


dose fluticasone increases the risk of URTI in patients with COPD, but not mometasone or budesonide.

\section{Supplementary information}

Supplementary information accompanies this paper at https://doi.org/10. 1186/s12890-020-01315-3.

Additional file 1. Search strategy.

\section{Abbreviations}

ICSs: Inhaled corticosteroids; URTI: Upper respiratory tract infection; COPD: Chronic obstructive pulmonary disease; RCTs: Randomized controlled trials; $\mathrm{FEV}_{1}$ : Forced expiratory volume in $1 \mathrm{~s}$; TORCH: The large prospective study Toward a Revolution in COPD Health; PRISMA: Preferred Reporting Items for Systematic Reviews and Meta-Analyses

\section{Acknowledgements}

Not applicable.

\section{Authors' contributions}

Study design: HC. Drafting of the manuscript: HC. Literature search: HC, YF and KW. Risk of bias assessment: HC, YF and KW. Statistical analysis: HC, YD, $\mathrm{KW}$ and JY. All authors read and approved the final manuscript.

\section{Funding}

This work was supported by the Chengdu Health Bureau Science and Technology Research Fund (Grant No. 2016013). The funding bodies had no role in the study design, data collection, analysis, and interpretation, or manuscript writing.

\section{Availability of data and materials}

The datasets used and/or analysed during the current study are available from the corresponding author on reasonable request.

\section{Ethics approval and consent to participate}

Not applicable.

\section{Consent for publication}

Not applicable.

\section{Competing interests}

The authors declare that they have no competing interests.

\section{Author details}

'Department of Infectious Disease, Chengdu Second People's Hospital, No. 10 Qingyun South Street, Chengdu 610017, China. ${ }^{2}$ Department of Respiratory Medicine and Critical Care Medicine, West China Hospital, Sichuan University, No. 37 Guo Xue Xiang, Chengdu 610041, China.

\section{Received: 17 January 2020 Accepted: 15 October 2020}

Published online: 28 October 2020

\section{References}

1. Global Initiative for Chronic Obstructive Lung Disease (GOLD). Global strategy for the diagnosis, management and prevention of COPD. http://www.goldcopd.org. Accessed 11 June 2018.

2. Mannino DM, Buist AS. Global burden of COPD: risk factors, prevalence, and future trends. Lancet. 2007;370(9589):765-73.

3. Vasily V. Global, regional, and national deaths, prevalence, disability-adjusted life years, and years lived with disability for chronic obstructive pulmonary disease and asthma, 1990-2015: a systematic analysis for the Global Burden of Disease Study 2015. Lancet Respir Med. 2017;5(9):691-706.

4. Spencer S, Calverley PM, Burge PS, Jones PW. Impact of preventing exacerbations on deterioration of health status in COPD. Eur Respir J. 2004; 23(5):698-702

5. Viniol C, Vogelmeier CF. Exacerbations of COPD. Eur Respir Rev. 2018; 27(147):170103.

6. Suissa S, McGhan R, Niewoehner D, Make B. Inhaled corticosteroids in chronic obstructive pulmonary disease. Proc Am Thorac Soc. 2007:4(7):535-42.
7. Lipworth BJ. Systemic adverse effects of inhaled corticosteroid therapy: a systematic review and meta-analysis. Arch Intern Med. 1999;159(9):941-55.

8. Suissa S, Kezouh A, Ernst P. Inhaled corticosteroids and the risks of diabetes onset and progression. Am J Med. 2010;123(11):1001-6.

9. Singh S, Amin AV, Loke YK. Long-term use of inhaled corticosteroids and the risk of pneumonia in chronic obstructive pulmonary disease: a metaanalysis. Arch Intern Med. 2009;169(3):219-29.

10. Cherry DK, Hing E, Woodwell DA, Rechtsteiner EA. National Ambulatory Medical Care Survey: 2006 summary. Natl Health Stat Report. 2008;(3):1-39. https://doi.org/10.1016/j.jaad.2004.07.067.

11. Calverley PM, Anderson JA, Celli B, Ferguson GT, Jenkins C, Jones PW, et al. Salmeterol and fluticasone propionate and survival in chronic obstructive pulmonary disease. N Engl J Med. 2007;356(8):775-89.

12. Sharafkhaneh A, Southard JG, Goldman M, Uryniak T, Martin UJ. Effect of budesonide/formoterol pMDI on COPD exacerbations: a double-blind, randomized study. Respir Med. 2012;106(2):257-68.

13. Mahler DA, Wire P, Horstman D, Chang CN, Yates J, Fischer T, et al. Effectiveness of fluticasone propionate and salmeterol combination delivered via the Diskus device in the treatment of chronic obstructive pulmonary disease. Am J Respir Crit Care Med. 2002;166(8):1084-91.

14. Kerwin EM, Scott-Wilson C, Sanford L, Rennard S, Agusti A, Barnes N, et al. A randomised trial of fluticasone furoate/vilanterol (50/25 $\mu$; 100/25 $\mu \mathrm{g})$ on lung function in COPD. Respir Med. 2013:107(4):560-9.

15. Vogelmeier CF, Bateman ED, Pallante J, Alagappan VK, Andrea P, Chen H, et al. Efficacy and safety of once-daily QVA149 compared with twice-daily salmeterol-fluticasone in patients with chronic obstructive pulmonary disease (ILLUMINATE): a randomised, double-blind, parallel group study. Lancet Respir Med. 2013;1(1):51-60.

16. Tashkin DP, Doherty DE, Kerwin E, Matiz-Bueno CE, Knorr B, Shekar T, et al, Efficacy and safety characteristics of mometasone furoate/formoterol fumarate fixed-dose combination in subjects with moderate to very severe COPD: findings from pooled analysis of two randomized, 52-week placebocontrolled trials. Int J Chron Obstruct Pulmon Dis. 2012;7:73-86. https://doi.org/10.2147/COPD.S29444.

17. Martinez FJ, Boscia J, Feldman G, Scott-Wilson C, Kilbride S, Fabbri L, et al. Fluticasone furoate/vilanterol $(100 / 25 ; 200 / 25 \mu \mathrm{g})$ improves lung function in COPD: a randomised trial. Respir Med. 2013;107(4):550-9.

18. Shamseer L, Moher D, Clarke M, Ghersi D, Liberati A, Petticrew M, et al. Preferred reporting items for systematic review and meta-analysis protocols (PRISMA-P) 2015: elaboration and explanation. BMJ. 2015;350:g7647.

19. Higgins JP, Altman DG, Gotzsche PC, Juni P, Moher D, Oxman AD, et al. The Cochrane Collaboration's tool for assessing risk of bias in randomised trials. BMJ. 2011:343:d5928.

20. Higgins JP. Thompson SG. Quantifying heterogeneity in a meta-analysis. Stat Med. 2002:21(11):1539-58.

21. Global INitiative for Asthma (GINA). Global strategy for asthma management and prevention. http://ginasthma.org. Accessed 18 May 2018.

22. Hanania NA, Darken P, Horstman D, Reisner C, Lee B, Davis S, et al. The efficacy and safety of fluticasone propionate (250 microg)/salmeterol (50 microg) combined in the Diskus inhaler for the treatment of COPD. Chest. 2003;124(3):834-43.

23. Zheng JP, Yang L, Wu YM, Chen P, Wen ZG, Huang WJ, et al. The efficacy and safety of combination salmeterol (50 microg)/fluticasone propionate (500 microg) inhalation twice daily via accuhaler in Chinese patients with COPD. Chest. 2007;132(6):1756-63.

24. Ferguson GT, Anzueto A, Fei R, Emmett A, Knobil K, Kalberg C. Effect of fluticasone propionate/salmeterol (250/50 microg) or salmeterol (50 microg) on COPD exacerbations. Respir Med. 2008;102(8):1099-108.

25. Calverley PM, Rennard S, Nelson HS, Karpel JP, Abbate EH, Stryszak P, et al. One-year treatment with mometasone furoate in chronic obstructive pulmonary disease. Respir Res. 2008;9:73.

26. Anzueto A, Ferguson GT, Feldman G, Chinsky K, Seibert A, Emmett A, et al. Effect of fluticasone propionate/salmeterol (250/50) on COPD exacerbations and impact on patient outcomes. COPD. 2009:6(5):320-9.

27. Calverley PM, Kuna P, Monsó E, Costantini M, Petruzzelli S, Sergio F, et al. Beclomethasone/formoterol in the management of COPD: a randomised controlled trial. Respir Med. 2010;104(12):1858-68.

28. Boscia JA, Pudi KK, Zvarich MT, Sanford L, Siederer SK Crim C. Effect of once-daily fluticasone furoate/vilanterol on 24-hour pulmonary function in patients with chronic obstructive pulmonary disease: a randomized, threeway, incomplete block, crossover study. Clin Ther. 2012;34(8):1655-66.e5. 
29. Doherty DE, Tashkin DP, Kerwin E, Knorr B, Shekar T, Banerjee S, et al. Effects of mometasone furoate/formoterol fumarate fixed-dose combination formulation on chronic obstructive pulmonary disease (COPD): results from a 52-week phase III trial in subjects with moderate-to-very severe COPD. Int J Chron Obstruct Pulmon Dis. 2012;7:57-71.

30. Tashkin DP, Doherty DE, Kerwin E, Matiz-Bueno CE, Knorr B, Shekar T, et al. Efficacy and safety of a fixed-dose combination of mometasone furoate and formoterol fumarate in subjects with moderate to very severe COPD: results from a 52-week phase III trial. Int J Chron Obstruct Pulmon Dis. 2012;7:43-55. https://doi.org/10.2147/COPD.S27319.

31. Huang K, Guo Y, Kang J, An L, Zheng Z, Ma L, et al. The efficacy of adding budesonide/formoterol to ipratropium plus theophylline in managing severe chronic obstructive pulmonary disease: an open-label, randomized study in China. Ther Adv Respir Dis. 2019;13:1753466619853500.

32. Suissa S, Baltzan M, Kremer R, Ernst P. Inhaled and nasal corticosteroid use and the risk of fracture. Am J Respir Crit Care Med. 2004;169(1):83-8.

33. Ni S, Fu Z, Zhao J, Liu H. Inhaled corticosteroids (ICS) and risk of mycobacterium in patients with chronic respiratory diseases: a metaanalysis. J Thorac Dis. 2014;6(7):971-8. https://doi.org/10.3978/j.issn.20721439.2014.07.03

34. Jing $X, L i Y, X U$ J. Risk of cardiovascular events associated with inhaled corticosteroid treatment in patients with chronic obstructive pulmonary disease: a meta-analysis. Can Respir J. 2018;2018:7097540.

35. Eichenhorn MS, Wise RA, Madhok TC, Gerald LB, Bailey WC, Tashkin DP, et al. Lack of long-term adverse adrenal effects from inhaled triamcinolone: lung health study II. Chest. 2003;124(1):57-62.

36. Highland KB. Inhaled corticosteroids in chronic obstructive pulmonary disease: is there a long-term benefit. Curr Opin Pulm Med. 2004;10(2):113-9.

37. Wolfe F, Caplan L, Michaud K. Treatment for rheumatoid arthritis and the risk of hospitalization for pneumonia: associations with prednisone, diseasemodifying antirheumatic drugs, and anti-tumor necrosis factor therapy. Arthritis Rheum. 2006;54(2):628-34.

38. Ek A, Larsson K, Siljerud S, Palmberg L. Fluticasone and budesonide inhibit cytokine release in human lung epithelial cells and alveolar macrophages. Allergy. 1999;54(7):691-9.

39. Belchamber KB, Thomas CM, Dunne AE, Barnes PJ, Donnelly LE. Comparison of fluticasone propionate and budesonide on COPD macrophage and neutrophil function. Int J Chron Obstruct Pulmon Dis. 2018;13:2883-97.

40. Yang $M$, Chen $H$, Zhang $Y, D u Y, X u Y$, Jiang $P$, et al. Long-term use of inhaled corticosteroids and risk of upper respiratory tract infection in chronic obstructive pulmonary disease: a meta-analysis. Inhal Toxicol. 2017; 29(5):219-26.

\section{Publisher's Note}

Springer Nature remains neutral with regard to jurisdictional claims in published maps and institutional affiliations.

Ready to submit your research? Choose BMC and benefit from:

- fast, convenient online submission

- thorough peer review by experienced researchers in your field

- rapid publication on acceptance

- support for research data, including large and complex data types

- gold Open Access which fosters wider collaboration and increased citations

- maximum visibility for your research: over $100 \mathrm{M}$ website views per year

At BMC, research is always in progress.

Learn more biomedcentral.com/submissions 